Portland State University

PDXScholar

Systems Science Faculty Publications and

Presentations

Systems Science

$7-2000$

\title{
Understanding Imperfection
}

Martin Zwick

Portland State University, zwick@pdx.edu

Follow this and additional works at: https://pdxscholar.library.pdx.edu/sysc_fac

Part of the Metaphysics Commons, and the Philosophy of Science Commons Let us know how access to this document benefits you.

Citation Details

Zwick, Martin, "Understanding Imperfection" (2000). Systems Science Faculty Publications and Presentations. 56.

https://pdxscholar.library.pdx.edu/sysc_fac/56

This Presentation is brought to you for free and open access. It has been accepted for inclusion in Systems Science Faculty Publications and Presentations by an authorized administrator of PDXScholar. Please contact us if we can make this document more accessible: pdxscholar@pdx.edu. 


\title{
UNDERSTANDING IMPERFECTION
}

\author{
Martin Zwick \\ Professor, Systems Science Ph.D. Program \\ Portland State University, Portland OR 97207-0751 \\ zwickm@pdx.edu http://www.sysc.pdx.edu/faculty/Zwick
}

Because of their inherent abstraction, systems ideas are not themselves sufficient for gaining scientific knowledge or solving practical problems, but they can be a source of insights into the universality of imperfection, insights which can contribute to a new scientific world view. Systems theory offers a metaphysics, or more precisely an ontology, of imperfection. Through it, we can heed Spinoza's injunction, "Not to lament, not to curse, but to understand."

$\underline{\text { Keywords: }}$ systems theory, metaphysics, ontology, imperfection, problems, insight

\section{Ideas Are for Insight}

In this talk, I want to present a conception that I have been working on for a number of years (Zwick, 1983, 1995) about the use of systems ideas. I start from the negative and proceed to the positive. The negative assertion is that systems ideas by themselves, unsupplemented by more specific and concrete knowledge (e.g., from the various disciplines), are insufficient for practical application, either for obtaining knowledge about the world or for solving problems. The reason for this is the inherent abstractness of these ideas. The positive assertion is that this metaphysics can be a rich source of insights needed to improve the human condition and a component of a new scientific world view.

The abstractness of systems ideas is succinctly captured by Bunge's characterization (1973) of the systems project as the attempt to construct an "exact and scientific metaphysics" (ESM). By metaphysics Bunge means general propositions about the world which hold for a wide variety of systems ("metaphysics" here thus does not refer to questions of the existence of God, free will, etc.). By scientific, he means grounded in, i.e., drawing upon and contributing to, the sciences. By exact, he means mathematical, or capable ultimately of being expressed mathematically (Bunge actually includes "exactness" within "scientific," but I pull it out as a separate idea). This ESM is presently constituted by a multiplicity of systems theories, e.g., information theory, control theory, game theory, and the like. A singular systems theory does not exist. In a sense, this is the goal of the systems project, a systems "theory of everything" (TOE) radically different from the more familiar TOE sought by physicists trying to unify the four fundamental forces. A systems "theory of everything" would be a true metaphysics, which would pertain to everything, not just the physical world and not merely in principle; it would touch every discipline, not merely physics. 
Here is a slightly different conceptualization: systems theories are less abstract than mathematics and philosophy but more abstract than theories in particular scientific disciplines (Boulding, 1956). By being more abstract than specific theories in the natural and social sciences, systems theories cannot without supplementary knowledge be evaluated empirically or used for practical purposes. Actually, even scientific theories are not directly testable. Theories, applied to different phenomena, offer models of these phenomena, and models, not their parent theories, are confronted by empirical evidence. Models are linked sets of relations, each a hypothesis which can be falsified, but models are not necessarily rejected by the failure of specific hypotheses; they may be rescued by plausible ad hoc adjustments. Finally, relations involve observables which define a "model object" appropriate to the phenomenon under study. This epistemological framework, based on Bunge's analysis, is summarized below in Table 1. Bunge does not include (2) as a distinct level, and I offer simpler names for the other levels. I add in italics the conception that systems theories are triadic in character (ESM), midway in abstraction between $\mathrm{E} \& \mathrm{M}$ and $\mathrm{S}$.

mathematics $(E)$

philosophy $(M)$

5. systems theory ["generic semi-interpreted theory"]

systems theories (ESM)

4. theory ["general theory"]

theories in the natural and social sciences $(S)$

3. model ["specific theory"]

2. relation, law, hypothesis

1. observables ["model object"]

Table 1. Epistemological hierarchy. [Bunge's terms are in brackets].

Models of phenomena (3) are produced either inductively from below by integrating observed regularities (2) or deductively from above by applying a theory (4) to a model object (1). Similarly (in italics), systems theories are produced inductively from below by identifying isomorphisms between different scientific theories $(\mathrm{S})$ or deductively from above by adding interpretations to mathematical formalisms (E) or concreteness (and exactness) to philosophical generalities (M).

This epistemological hierarchy suggests that unless systems-theoretic notions are used in specific scientific theories and more explicitly in models of particular phenomena, they are too far removed from the real-world to offer usable knowledge by themselves. For example, game theory does not explain behavior observed in auctions and cannot guide rational action in such settings, but if supplemented by additional information about economic systems in general and about auctions in particular, game theory could provide the basis for a descriptive and/or normative theory of auction behavior. 
However, while an economist might draw upon game-theory to develop a model of auctions, such a model is unlikely to be comprehensible to scientists in other fields, to other scholars, and to the public at large. For the general public, the scientific study of particular phenomena is rarely feasible. It might seem therefor that the project of constructing an "exact and scientific metaphysics" is irrelevant except to scientists.

This is not so. What non-specialists need to navigate the many worldly contexts which they encounter is insight. Insight is more usable than theory or methodology because it is flexible and open-ended. Insight is what a metaphysics can offer. The significance of the systems project, aimed at a metaphysics which is precise and grounded, may well consist more in its general cultural impact than its scientific fecundity. This is the positive aspect of the deep abstraction which characterizes systems ideas.

\section{Insight is Needed into Imperfection}

What is not problematic does not require understanding. It is imperfection which generates the need for insight. In the social world, "imperfection" means war, injustice, exploitation, and the like. Speaking more narrowly about culture, our values are imperfect not merely in the difficulty of realizing them, but because the values themselves are contradictory. This is the anti-utopian position of Isaiah Berlin (1991) who argues that cultural values do not resemble the mutually consistent axioms of a formal mathematical system, but are typically incompatible. In the natural world, "imperfection" encompasses the dangers arising in the environment to human beings and other organisms and, reciprocally, the local and global threats to the environment posed by human action. In the biological world, "imperfection" includes disease, suffering, and (from the vantage point of prey) predation. In the psychological and behavioral worlds, there are imperfections in our characters and our actions. And so on. One need not belabor the obvious. Imperfection abounds in the natural and social worlds. In so far as we must deal with it, we need insight into its deepest, "metaphysical," basis.

I am not speaking of imperfection in our models of the world, but in the world itself. That is, I want to discuss ontology and not epistemology, and employ an objective rather than a subjective mode of discourse. Imperfections in models can be included while retaining an objective stance by considering deficiencies in the "modeling subsystems" of organisms, organizations, and societies.

To say that imperfection is objective is not to deny that imperfection implies the vantage point of agents who have interests. To a first approximation, we humans are the reference point, as we are the ones making judgments. But the reference point is actually larger and includes all forms of life. Every living system has interests and in so far as such a system can directly or indirectly further its interests, it exhibits agency. To make the notion of "interests" exact, one can define for a system a utility function. Systems having interests is the subject of game theory, in which utility is given formal treatment (von Neumann \& Morgenstern, 1944). Utility with respect to reproduction is "fitness," hence the use of game-theory in evolutionary biology, but utility is a broader notion. 
From a biological perspective, the title of this essay might have referred to understanding "vulnerability" or "hazard," but "imperfection" is plainer and more general.

From the perspective of individual living systems, the universe is less than perfect. Instead of speaking of imperfection, one could speak of "problems" which we or other organisms face, but the word "imperfection" has the virtue of suggesting an objective character to these problems. Imperfection is not merely in the eye of the beholder. Problems reflect a mismatch between what is actual and what is ideal to some agent, and what is ideal may be subjective, but it may alternatively be objective. The survival needs of organisms are objective facts. More importantly, the causes of the mismatch between ideal and actual are objective features of the world, and many of these features are ubiquitous, perhaps even universal. This is a Gnostic or Kabbalist view. Imperfection is pervasive, and an exact and scientific metaphysics would lay bare its most general, i.e., ontological, foundations. To regard imperfection as a metaphysical condition, however, does not mean that this condition is irremediable, just that problems are lawful and ubiquitous. Perfection is precluded by limitation, and limitation is the precondition of existence. What remains possible is perfecting, the verb, which is better anyway than perfection, the noun, a state which is unattainable, because only the process and not the state produces the drama of history, the absence of whose performance would surely be an imperfection.

I want to make several qualifying remarks concerning this point of view. First, let me mention the position, more popular in the past, articulated for example by Leibniz and mocked by Voltaire, that everything is perfect just as it is. It is hard to imagine a 21st century writer who would find such a position still worthy of parody. Even were this true at some "high level of reality," at the level we live on, it is untrue. Moreover, even the highest truth is not the whole truth, and the whole takes precedence over the highest. From a religious, or, more precisely, a doctrinal perspective, the pervasiveness of imperfection raises the theological problem of theodicy, i.e., the conundrum of how there could be a God who is all-knowing, all-powerful, and all-good, given the evil and suffering in the world. A systems metaphysics, being necessarily silent about the existence of God, cannot solve this conundrum, but the "secular theodicy" which such a metaphysics can offer might be a valuable corrective to the Leibnizian optimism of current anthropic speculation.

Finally, I want to acknowledge the negativity which afflicts a focus on imperfection. A glass half empty is also half-full, so the word "imperfection" is itself imperfect in its exclusively negative connotation. Imperfection necessarily encompasses the possibility of perfecting. I mean actually to assert something more like a mixture of perfection and imperfection or a delicate balance between the two, perhaps something like the idea of the "edge of chaos" (Langton, 1992). 


\section{Systems Ideas about Imperfection}

There are many systems ideas and themes which bear on the subject of imperfection and what follows is a only a sample.

- Tensions, not restricted to formal mathematical systems, between incompleteness and inconsistency

- Imbalances involving other fundamental dualities, such as variety and constraint, order and disorder, unity and multiplicity

- Problems of boundary, e.g., the issue of crisp vs. fuzzy boundaries, excessive or inappropriate openness or closedness

- Instability, the destruction of order through chaos or catastrophe; or, conversely: overstability, rigidification

- Pathologies of (feedback/feedforward) control

- Aberrations of hierarchical order, including the opposing needs of centralization and decentralization, differentiation and integration

- Informational parasitism (internal or external)

- Dependence upon and constraint by the environment

- Limitations of agency: multiple deciders or objectives (inconsistency) or uncertainty (incompleteness), problems of global optimization, computational intractability, and undecidability; unintended consequences and counter-intuitive effects

- Difficulties presented by other systems; competition, predation, exploitation; tensions between autonomy and interdependence; dilemmas involving multiple agents

- Embeddedness in and thus control by more encompassing systems

- Impermanence and fragility of order; vulnerability to events on smaller, larger, or the same scale

- Limitations of modeling subsystems: the inherent indistinguishability of variety and noise, of reality and illusion

This is just a list of systems ideas relevant to the theme of imperfection. If one tabulated, instead of ideas, the theories which bear on this subject, one would include most of the well-developed theories central to the systems project, e.g., information theory, graph theory, dynamic systems theory, control theory, game theory, non-equilibrium thermodynamics, evolutionary theory, etc. For convenience in this essay, I use the name "Problematics" as a working label for the enterprise of collecting, formulating, and organizing systems ideas about imperfection. In earlier work (Zwick, 1983), I tried to weave together such ideas into a metaphysical narrative about imperfection. This essay and its precurser (Zwick, 1995) explain the motivation for this undertaking.

The ideas on this list, and other related ideas, can be given exact expression and linked to current scientific knowledge. There is thus the possibility of an ontology of problems, a systems metaphysics organized around the theme of imperfection. But here we come face to face with the imperfection of systems theory itself. As already noted, a unitary theory does not exist; instead we have a multiplicity of theories, weakly linked to one another. Some means must still be found to bring order and unity to this multiplicity. 
I suspect what is needed for this is something like the hierarchy of systems types sketched by Boulding (1956): (i) frameworks, (ii) clockworks, (iii) control (cybernetic) systems, (iv) open systems self-maintained by matter-energy throughput, (v) genetic-societal systems (plants), (vi) purposeful information-processing systems (animals), (vii) selfconscious symbol-processing systems (humans), and, not at all discussed by Boulding, (ix) "transcendental" systems. Such a hierarchy brings the contemporary idea of "complexity" into proximity with the traditional idea of "being." The notion of complexity, while capable of being interpreted in many different ways, can be made exact and scientific, but the notion of being is metaphysically more substantial. From the perspective of the construction of an exact and scientific metaphysics, it is interesting and probably not accidental that Boulding's hierarchy evokes -- even resembles -- the religious-philosophical idea of the "great chain of being" (Lovejoy, 1936), which was widely accepted in Western culture though most of its history. (This traditional idea also directly addressed the ubiquity of imperfection, viewing it as a necessary part of a greater perfection.) With Boulding's proposal as a first approximation, one can at least imagine a systems-theoretic version of "the great chain" which would satisfy the dual requirements of being exact and scientific. No doubt some of the assumptions (e.g., the principles of continuity and plenitude) which characterized the traditional idea would have to be discarded or altered. As the "skeleton" of an ESM, a modern "chain of complexity" would probably have to be discrete with a modest number of levels, and would have to accept contingency, anathema to traditional doctrine, as fundamentally implicated in the creative generativity of nature.

Why would one undertake to construct a systems-theoretic ontology of imperfection? The motivation is deeper than the mere solution of problems. Metaphysics is a basis for morals (Murdoch, 1992). Spinoza for example begins his Ethics (1677) with a systems metaphysics (Jonas, 1965). This approach was natural in the premodern era: one begins with a description of the world and then considers how one should live in it; one moves from "is" to "ought." Philosophers since Hume have however pointed out that one cannot derive "ought" from "is." True enough: fact by itself is not sufficient, but it is necessary. Though domains of fact and value may be sharply distinguished, they interpenetrate in a complex manner, like two basins of attraction of a nonlinear dynamic system whose separating boundary is fractal. Moreover, value emerges in a world of fact. The existence of value is a fact. The notion of utility, developed in game theory and grounded in biology and the social sciences, bridges the two domains. (More generally, game theory itself, being capable of both descriptive and normative use, exhibits the interconnection of fact and value.) The formal idea of utility is much richer than its colloquial connotation of usefulness, which is largely external in character: utility might also quantify value internal to a system, i.e., perfection of structure. The notion of utility augments those of matter, energy, and information as a fourth fundamental category, instantiated and visible in the phenomena of life. It is a notion which can encompass both the objective and the subjective, both the necessary and the contingent; on the human scale, both needs and wants. 
Without understanding what is, one really cannot consider what ought to be, at least not effectively, so metaphysics bears significantly on ethics. Game theory, for example, brings exactness and empirical knowledge into contact with ethics. From insight into the ontology of imperfection there follows the possibility and thus the responsibility of perfecting, of "tikkun," to use a term from Kabbalist metaphysics meaning the fixing of metaphysical imperfection as far as is possible. Or, as the philosopher Levinas (1989) has argued, one should even reverse the sequence and say that ethics precedes ontology because it motivates -- or should motivate -- metaphysics, as it does for Spinoza.

"Problematics," that is, systems inquiry into the origins and nature of imperfection is not sufficient. Problematics is less than "diagnostics" -- being able to say what the critical issues are in any particular problem. One might imagine a physician's desk manual (a DSM-V) for the diagnosis of metaphysical ills. Diagnostics in turn falls short of "therapeutics" -- knowledge of what to do about the problem, of how perfecting might be accomplished. But the progression from problematics to diagnostics to therapeutics, however important, is not to be rushed. Facile diagnostics and simplistic therapeutics are often counterproductive, this being one of the lessons gained from systems thinking. The assumption that we already understand problems adequately is usually illusory. What is needed most to solve problems is not theory or methodology but insight, if it is deep and especially if it is widely shared.

\section{The Contribution of Systems Ideas}

A metaphysics of imperfection would contribute to a new scientific world view which is coherent and moral. In a reductionist world view, there is no imperfection, only fundamental particles and their interactions, and life is a mere epiphenomenon. Systems thought offers a radically different view. It replaces the hegemony of the fundamental with the priority of the central, and hence of the general. From the perspective of the central, all systems are ontologically equal (though from the perspective of "complexity" -- or "being" -- they are not). Ultimately, also, all are flawed by limitation, which is the price of existence. To use Murdoch's phrase (1992), systems are "partial wholes" [emphasis added]. Again, it is important to point to the virtue in the flaw. In the words of the poet Leonard Cohen, "there is a crack in everything; this is to let the light in."

A systems metaphysics can be a bridge from science to the humanities, the arts, philosophy, religion. There was once, from the medieval period to the beginnings of modern science, an integrated view of matter, life, mind, cosmos. This view suffered, however, from an overvaluation of order and harmony, from being inexact and empirically undeveloped, and from being tied to one religious tradition. It eventually disintegrated with the rise of science, leaving a cultural vacuum described poignantly in Yeats' complaint, “...all coherence lost...”. Post-modern nihilism may be the final act of this decline, but other developments hint at the possibility of a recovery of cultural coherence, of a new integrated world view centered in science (Toulmin, 1982) but open to other realms of human experience. The project of constructing an exact and scientific metaphysics, however fragmented and fragile an undertaking, is a vital part of this possibility. 
It is significant also that systems ideas can contribute not only to culture at large but to "personal knowledge" (Polanyi, 1964), which can be individually appropriated and used in our private lives. Because the systems view does not reduce, because it gives priority to form (which we perceive) over matter (about which our direct experience is limited), because it seeks cross-level truths (applicable "above" to natural and social systems, and "below" to ourselves), and because it is not committed solely to 3rd person objectivity but encompasses also 1st person subjectivity, for these and other reasons, systems ideas, unlike most ideas in science, can be meaningful to us. They might even help us understand the lawfulness but not hopelessness of our own imperfections.

To close the circle of this argument (and perhaps return to the negative): the strength of an exact and scientific metaphysics is also its weakness. The main obstacle preventing systems ideas from contributing significantly to a new scientific world view and to personal knowledge is the abstractness of these ideas, the very abstractness from which their scope and significance derives. This abstractness limits the number of people who become aware of systems ideas. It also tends to restrict the understanding of these ideas to a strictly intellectual mode, which lacks the emotional force needed to change our image of and relation to the world. This poses an important educational challenge, but that is another subject.

\section{References}

Berlin, I., edited by H. Hardy (1991). The Crooked Timber of Humanity. New York: Alfred A. Knopf.

Boulding, K. (1956). "General Systems Theory - the Skeleton of Science." Management Science 2, pp. 197-208. Reprinted in Buckley, ed. (1968), Modern Systems Research for the Behavioral Scientist. Chicago: Aldine, pp. 3-10.

Bunge, M. (1973). Method, Model, and Matter. Boston: D. Reidel.

Jonas, Hans (1965). "Spinoza and the Theory of the Organism." Journal of the History of Philosophy, 3, pp. 43-58. Also reprinted in Grene, Marjorie, ed. (1979). Spinoza. A Collection of Critical Essays. Notre Dame: University of Notre Dame Press, pp. 259278.

Langton, C. (1992). "Life at the Edge of Chaos." Artificial Life II. SFI Studies in the Sciences of Complexity, vol. X, ed. by C.G. Langton, C. Taylor, J.D. Farmer, \& S. Rassmussen, Redwood City CA: Addison-Wesley.

Levinas, E. (1989). “Is Ontology Fundamental?” Philosophy Today, Summer 1989, p.127.

Lovejoy, A. (1936). The Great Chain of Being. Cambridge: Harvard University Press. 
Murdoch, I. (1992). Metaphysics As a Guide for Morals. London: Penguin.

von Neumann, J., \& Morgenstern, O. (1944). Theory of Games and Economic Behavior. New York: Wiley.

Polanyi, M. (1964). Personal Knowledge. New York: Harper and Row.

Spinoza, B. (1677), translated by A. Boyle (1967). Ethics. New York: Dutton.

Toulmin, S. (1982). The Return to Cosmology. Berkeley: University of California Press.

Zwick, M. (1983). "Incompleteness, Negation, Hazard: On the Precariousness of Systems," The Relation Between Major World Problems and Systems Learning, Vol. 1, Proceedings of the 1983 Annual Conference of the Society for General Systems Research, Intersystems, California, 197-203; reprinted in Nature and System, 6, 33-42, 1984.

Zwick, M. (1995). "Towards an Ontology of Problems." Advances in Systems Science and Applications Special Issue I, pp. 37-42. 